\title{
Letter
}

\section{The ground state of the Pomeron and its decays to light mesons and photons}

\author{
A. A. Godizov ${ }^{\mathrm{a}}$ \\ SRC Institute for High Energy Physics of NRC "Kurchatov Institute”, 142281 Protvino, Russia
}

Received: 9 May 2016 / Accepted: 23 June 2016 / Published online: 30 June 2016

(C) The Author(s) 2016. This article is published with open access at Springerlink.com

\begin{abstract}
The problem of the timelike Pomeron coupling to light mesons and photons is considered in light of available data on high-energy meson-proton scattering. A possible correspondence of the $f_{2}$ (1950) resonance to the ground state of the Pomeron is argued.
\end{abstract}

\section{Introduction}

In Regge phenomenology [1], the Pomeron is introduced as an even Reggeon with vacuum quantum numbers and the intercept of its Regge trajectory higher than unity. Any Reggeon is defined as an analytic continuation of some set of bound states and resonances to the region of complex values of the spin. Many meson Reggeons emerging in the Quark Model have already got their identification via association with experimentally observed hadron states [2]: $f$-Reggeon ( $f_{2}(1270)$ and $f_{4}(2050)$ mesons), $\rho$-Reggeon $(\rho(770)$ and $\rho_{3}$ (1690) mesons), $a$-Reggeon, $\omega$-Reggeon, $\phi$-Reggeon, $\pi$ Reggeon, etc. All meson Regge trajectories have intercepts noticeably lower than unity. As a consequence, high-energy diffractive scattering of hadrons is dominated by Pomeron interaction. In particular, the representation of elastic scattering amplitude in terms of Pomeron exchanges yields a satisfactory description of the nucleon-nucleon diffractive pattern at ultrahigh collision energies and low momentum transfers [3]. However, being one of the most important objects of strong interaction physics (the main carrier of the strong interaction in the high-energy diffraction domain), the Pomeron has not been discovered yet. The question "which of the observed $0^{+}\left(2^{++}\right)$states, if any, is the ground state of the Pomeron?" still waits for proper answer.

From the practical standpoint, true identification of the Pomeron is possible only through estimation of the partial decay widths and branching ratios of its real states (even-spin resonances). Presumably, the Pomeron resonance decays to

a e-mail: anton.godizov@gmail.com "hadron-antihadron" pairs are driven by the same functions as the Pomeron coupling to the corresponding hadrons in high-energy diffractive scattering (see the appendix). If we knew the analytic structure of these functions, we would be able to calculate the partial decay widths of the Pomeron real states. Unfortunately, at its present stage of development, QCD does not help to solve the problem. Nevertheless, some relevant information can be extracted from the available experimental data on meson-proton scattering. In this paper, we will make an attempt to identify the ground state of the Pomeron, searching for interrelation between the high-energy hadron diffraction data and the measured characteristics of observed $0^{+}\left(2^{++}\right)$resonances.

For example, in the framework of the DL model [4], the ratio of the kaon-proton and pion-proton total cross sections is approximately equal (up to secondary Reggeon contributions) to the ratio of the Pomeron couplings to the corresponding mesons in forward scattering. At $\sqrt{s}=24.1 \mathrm{GeV}$ (the highest reached energy for $K p$ scattering), the impact of secondary Reggeons on the quantity [5]

$\frac{\sigma_{\text {tot }}^{K^{+} p}}{\sigma_{\text {tot }}^{\pi^{+} p}} \approx 0.844$

is minimal. As the partial decay width of the Pomeron ground state to pair of scalar mesons is proportional to the squared absolute value of the corresponding meson-Pomeron coupling, so, using (1), we obtain a qualitative estimation

$\frac{\Gamma_{\mathrm{P} \rightarrow K^{+} K^{-}}}{\Gamma_{\mathrm{P} \rightarrow \pi^{+} \pi^{-}}} \sim 0.844^{2} \approx 0.71$.

This value is consistent with the $f_{2}(1950)$ resonance branching ratio

$\frac{\Gamma_{f_{2}(1950) \rightarrow K^{+} K^{-}}}{\Gamma_{f_{2}(1950) \rightarrow \pi^{+} \pi^{-}}}=0.565_{-0.27}^{+0.19}$

extracted from the Belle Collaboration data on the quantities $\Gamma(\gamma \gamma) \Gamma\left(\pi^{0} \pi^{0}\right) / \Gamma_{\text {tot }}[6]$ and $\Gamma(\gamma \gamma) \Gamma\left(K^{+} K^{-}\right) / \Gamma_{\text {tot }}[7]$. 
On the one hand, such a proportion between the partial decay widths could be considered characteristic for any $S U(3)_{f}$ singlet. On the other hand, in the $t-J$ plane, $f_{2}(1950)$ resonance is located very close to the DL Pomeron Regge trajectory, $\alpha_{\mathrm{P}}^{D L}(t)=1.08+0.25 t[8,9]$, widely used in phenomenology. Therefore, it may be considered as a promising candidate for the Pomeron ground state.

However, $0^{+}\left(2^{++}\right)$hadrons different from $f_{2}(1950)$ should not immediately be excluded from the consideration. Of course, the observed smallness of the branching ratios $\frac{\Gamma_{f_{2}(1270) \rightarrow K \bar{K}}}{\Gamma_{f_{2}(1270) \rightarrow \pi \pi}}$ and $\frac{\Gamma_{f_{2}^{\prime}(1525) \rightarrow \pi \pi}}{\Gamma_{f_{2}^{\prime}(1525) \rightarrow K \bar{K}}}$ [2] allows one to consider resonances $f_{2}(1270)$ and $f_{2}^{\prime}(1525)$ as mesons of certain quark-antiquark content and to relate them to secondary Reggeons, but the current interpretation of other well-established $0^{+}\left(2^{++}\right)$resonances (namely, $f_{2}(2010)$, $f_{2}(2300)$, and $f_{2}(2340)$ [2]) is not so clear. To distinguish reliably the Pomeron spin-2 state among various vacuum resonances of the same spin, we need more information on the Pomeron dynamics in both the spacelike and the domains. First, let us estimate the Pomeron coupling to light mesons in the diffractive scattering regime.

\section{The spacelike Pomeron coupling to light mesons}

\subsection{Elastic scattering of scalar mesons on protons}

In the leading approximation, the angular distributions in $\pi^{+} p$ and $K^{+} p$ elastic scattering can be calculated in the same way as for $p p$ scattering (see [3] and references therein):

$$
\begin{aligned}
& \frac{\mathrm{d} \sigma}{\mathrm{d} t}=\frac{|T(s, t)|^{2}}{16 \pi s^{2}}, \\
& T(s, t)=4 \pi s \int_{0}^{\infty} \mathrm{d} b^{2} J_{0}(b \sqrt{-t}) \frac{e^{2 i \delta(s, b)}-1}{2 i}, \\
& \delta(s, b)=\frac{1}{16 \pi s} \int_{0}^{\infty} \mathrm{d}(-t) J_{0}(b \sqrt{-t}) \delta_{\mathrm{P}}(s, t) \\
& \quad \approx \frac{1}{16 \pi s} \int_{0}^{\infty} \mathrm{d}(-t) J_{0}(b \sqrt{-t}) g_{h h \mathrm{P}}(t) g_{p p \mathrm{P}}(t) \\
& \quad \times\left(i+\tan \frac{\pi\left(\alpha_{\mathrm{P}}(t)-1\right)}{2}\right) \pi \alpha_{\mathrm{P}}^{\prime}(t)\left(\frac{s}{2 s_{0}}\right)^{\alpha_{\mathrm{P}}(t)},
\end{aligned}
$$

where $s$ and $t$ are the Mandelstam variables, $b$ is the impact parameter, $s_{0}=1 \mathrm{GeV}^{2}, \alpha_{\mathrm{P}}(t)$ is the Regge trajectory of the Pomeron, $g_{p p \mathrm{P}}(t)$ is the Pomeron coupling to the proton, and $g_{h h \mathrm{P}}(t)$ is the Pomeron coupling to the incoming meson. At $t<0$, the Pomeron Regge trajectory and the Pomeron coupling to the proton are approximated by the same test functions as in nucleon-nucleon scattering [3],

$$
\alpha_{\mathrm{P}}(t)=1+\frac{\alpha_{\mathrm{P}}(0)-1}{1-\frac{t}{\tau_{a}}}, \quad g_{p p \mathrm{P}}(t)=\frac{g_{p p \mathrm{P}}(0)}{\left(1-a_{g} t\right)^{2}},
$$

Table 1 The parameter values for (5) fitted to the nucleon-nucleon elastic scattering data

\begin{tabular}{ll}
\hline Parameter & Value \\
\hline$\alpha_{\mathrm{P}}(0)-1$ & $0.109 \pm 0.017$ \\
$\tau_{a}$ & $(0.535 \pm 0.057) \mathrm{GeV}^{2}$ \\
$g_{p p \mathrm{P}}(0)$ & $(13.8 \pm 2.3) \mathrm{GeV}$ \\
$a_{g}$ & $(0.23 \pm 0.07) \mathrm{GeV}^{-2}$ \\
\hline
\end{tabular}

where the free parameters take on the values presented in Table 1.

Such a choice of parametrization for $\alpha_{\mathrm{P}}(t)$ is in part specified by the QCD-motivated asymptotic behavior of the Pomeron Regge trajectory [10],

$\lim _{t \rightarrow-\infty} \alpha_{\mathrm{P}}(t)=1$,

and by the conditions

$\frac{\mathrm{d}^{n} \alpha(t)}{\mathrm{d} t^{n}}>0 \quad(n=1,2 \ldots ; t<0)$,

which originate from the dispersion relations for Regge trajectories [1] and are expected to be valid for any Reggeon. ${ }^{1}$

Fixing

$g_{\pi \pi \mathrm{P}}(t)=g_{\pi \pi \mathrm{P}}(0)=8.0 \mathrm{GeV}$

and

$g_{K K \mathrm{P}}(t)=g_{K K \mathrm{P}}(0)=7.1 \mathrm{GeV}$,

we come to the description presented in Fig. 1 (the solid lines). The secondary Reggeon interaction influence on the $\pi^{+} p$ and $K^{+} p$ total cross sections can be roughly taken into account via straight addition of the corresponding DL model terms [4] (see the dashed lines in Fig. 1):

$$
\begin{aligned}
& \Delta \sigma_{\text {tot }}^{\pi^{+} p}=27.56\left(\frac{s}{s_{0}}\right)^{-0.4525} \mathrm{mb}, \\
& \Delta \sigma_{\text {tot }}^{K^{+} p}=8.15\left(\frac{s}{s_{0}}\right)^{-0.4525} \mathrm{mb} .
\end{aligned}
$$

The deviation of the model differential cross section curves from the experimental data cannot be removed so easily, since it requires introduction of extra unknown functions of $t$. The main problem is that, in view of the rather high slopes of the secondary Regge trajectories in the resonance region,

$\alpha_{\mathrm{R}}^{\prime}(t) \sim 1 \mathrm{GeV}^{-2} \quad(t>0)$,

\footnotetext{
${ }^{1}$ Other analytic properties of parametrizations (5) in no way deserve serious consideration. These expressions should be treated just as some nonanalytic quantitative approximations (valid at low negative $t$ only) to the corresponding true dynamic functions whose analytic structure is still unknown.
} 


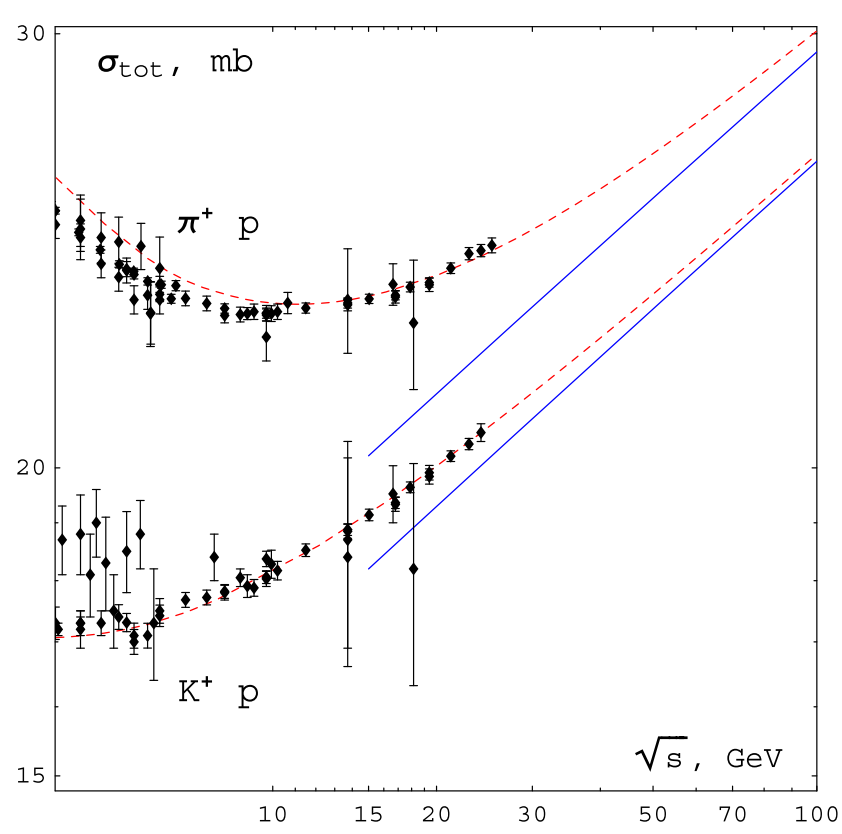

Fig. 1 The model description of the $\pi^{+} p$ and $K^{+} p$ scattering observables $[5,11]$. The solid lines correspond to the Pomeron exchange eikonal approximation (4), (5) of the amplitude. The dashed lines are

their intercept values,

$\alpha_{\mathrm{R}}(0) \sim 0.5$,

their asymptotic behavior in the deeply Euclidean domain $[12,13]$,

$\lim _{t \rightarrow-\infty} \alpha_{\mathrm{R}}(t)=0$,

and conditions (7), they are expected to be essentially nonlinear in the diffractive scattering range. Hence, $t$-behavior of the corresponding Regge residues turns out strongly related to the nontrivial $t$-behavior of the factors $\alpha_{\mathrm{R}}^{\prime}(t)$ which originate from the Reggeon resonance propagators (for details, see the appendix of [3]). As a consequence, reliable determination of the secondary Reggeon exchange contributions into the eikonal (Born amplitude) at nonzero $t$ is possible only via combined fitting of all the unknown functions to the overall set of available data on nucleon-nucleon and meson-proton scattering, including charge exchange reactions [14-16]. Such a statistically satisfactory description is not achieved yet. It still remains one of the most crucial challenges in Regge phenomenology.

Nonetheless, the relative divergence between the presented model curves and the data is limited. The underestimation of $|T(s, t)|$ at $\sqrt{s}=21.7 \mathrm{GeV}$ does not exceed 16 and $25 \%$ for the $\pi^{+} p$ and $K^{+} p$ scattering, respectively. ${ }^{2}$

\footnotetext{
${ }^{2}$ In particular, the model estimation of the $\pi^{+} p$ differential cross section slope at $t=-0.2 \mathrm{GeV}^{2}, B^{\text {model }} \approx 8.7 \mathrm{GeV}^{-2}$, coincides with the measured value (see Fig. 2 in [11]).
}

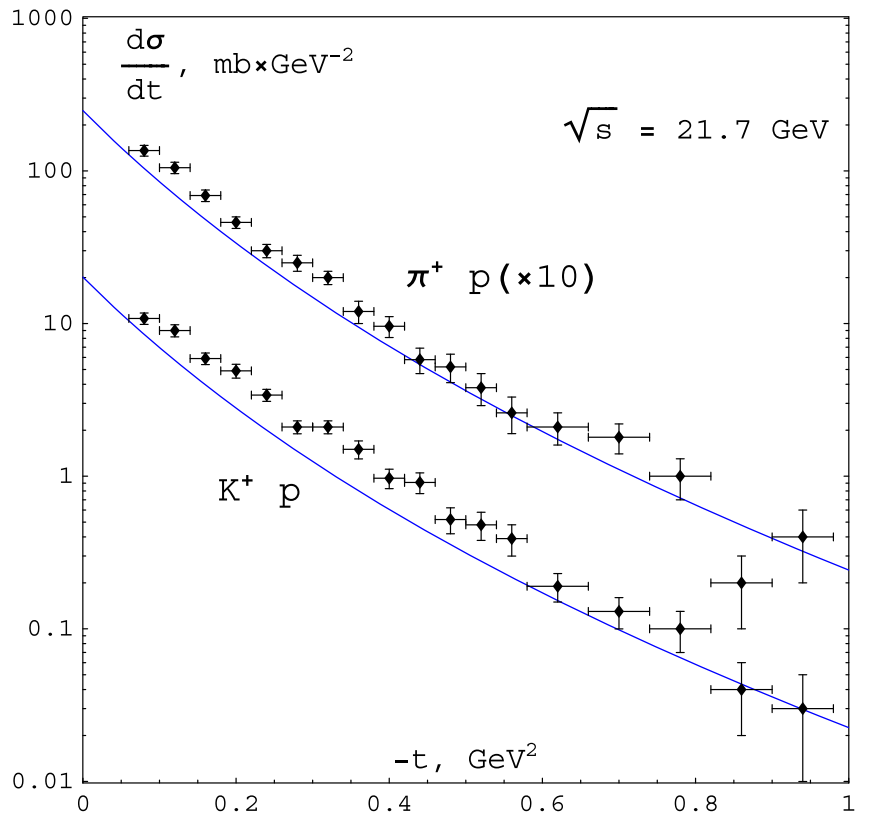

obtained by adding the secondary Regge pole terms (10) of the DL model for total cross sections [4]

This fact might be interpreted as a hint to rather weak $t$ dependence of the Pomeron coupling to light scalar mesons in the diffractive scattering regime. Unfortunately, the contamination by secondary Reggeon exchanges (though subdominant) does not allow one to establish this $t$-behavior explicitly in the framework of approximation (4). To elucidate on this matter, we need to analyze available data on the high-energy scattering of vector mesons on protons.

\subsection{Exclusive photoproduction of vector mesons on protons}

The vector meson photoproduction data [17-24] are available in the kinematic range wherein the influence of secondary Reggeon exchanges is negligible. According to the hypothesis of $s$-channel helicity conservation (SCHC), confirmed experimentally [25-28], the cross sections of reactions $\gamma+p \rightarrow V+p$ are dominated by the non-flip helicity amplitudes. In the leading approximation, these amplitudes can be represented as $[29,30]$

$T_{\gamma p \rightarrow V p}\left(W^{2}, t\right) \approx \sum_{V^{\prime}} C_{T}^{V^{\prime}}(0) T_{V^{\prime} p \rightarrow V p}\left(W^{2}, t\right)$,

where $W$ is the collision energy and $C_{T}^{V}(0)$ are the vector dominance model (VDM) coefficients:

$C_{T}^{V}\left(Q^{2}\right)=\sqrt{\frac{3 \Gamma_{V \rightarrow e^{+} e^{-}}}{\alpha_{e} M_{V}}} \frac{M_{V}^{2}}{M_{V}^{2}+Q^{2}}$

( $M_{V}$ is the vector meson mass and $Q^{2} \ll M_{V}^{2}$ is the photon virtuality). It is well known that at small values of $t$ the 

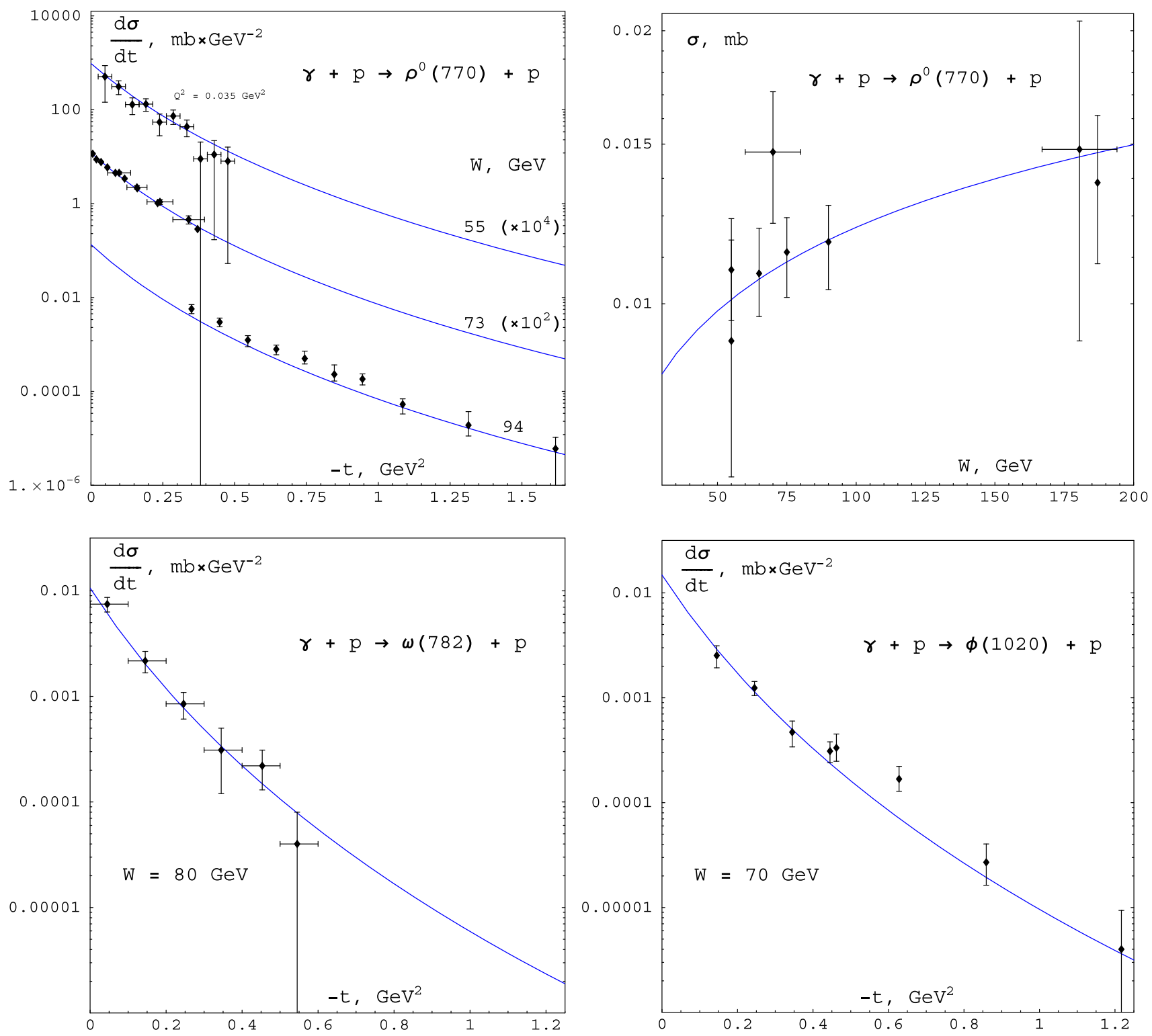

Fig. 2 The model description of the vector meson photoproduction observables [17-24]

Pomeron diagonal coupling to hadrons is much stronger than the off-diagonal one. Otherwise, say, the cross section of the low-mass diffractive excitation of proton in high-energy $p p$ collisions would be comparable to the elastic cross section. Thus, in the range $W>30 \mathrm{GeV}$,

$T_{\gamma p \rightarrow V p}\left(W^{2}, t\right) \approx C_{T}^{V}(0) T_{V p \rightarrow V p}\left(W^{2}, t\right)$,

where $T_{V p \rightarrow V p}\left(W^{2}, t\right)$ is calculated with the help of (4).

Fixing

$$
\begin{aligned}
& g_{\omega \omega \mathrm{P}}(t)=g_{\rho \rho \mathrm{P}}(t)=g_{\rho \rho \mathrm{P}}(0)=7.07 \mathrm{GeV}, \\
& g_{\phi \phi \mathrm{P}}(t)=g_{\phi \phi \mathrm{P}}(0)=6.7 \mathrm{GeV},
\end{aligned}
$$

we come to the description presented in Fig. 2 and Table 2. The predicted exponential slope of $\mathrm{d} \sigma / \mathrm{d} t$ for $\gamma+p \rightarrow$ $\rho^{0}(770)+p$ at $W=73 \mathrm{GeV}$ and $0.073 \mathrm{GeV}^{2}<-t<$ $0.4 \mathrm{GeV}^{2}, B^{\text {model }} \approx 9.1 \mathrm{GeV}^{-2}$, is compatible with the measured value, $B^{\exp }=(9.8 \pm 1.36) \mathrm{GeV}^{-2}[20]$.

The deviation of the $\rho^{0}(770)$ and $\phi(1020)$ photoproduction curves from the data at $-t>0.4 \mathrm{GeV}^{2}$ could be explained by the $15 \%$ normalization uncertainty [22] not included in the error bars. If we multiply the $\rho^{0}(770)$ photoproduction data set [22] by factor 0.85 and exclude two outlying points, at $t=-0.019 \mathrm{GeV}^{2}$ and $t=$ $-0.371 \mathrm{GeV}^{2}$, from data set [21], the description quality becomes much better: $\chi^{2} / N_{D o F} \approx 1.17$ (37 points, one free parameter).

Fitting the $t$-dependent test expressions of the $\rho^{0}(770)$ Pomeron coupling, 
Table 2 The quality of description of the experimental angular distributions in the vector meson exclusive photoproduction on protons at $g_{\omega \omega \mathrm{P}}(t)=g_{\rho \rho \mathrm{P}}(t)=g_{\rho \rho \mathrm{P}}(0)=7.07 \mathrm{GeV}$ and $g_{\phi \phi \mathrm{P}}(t)=g_{\phi \phi \mathrm{P}}(0)=$ $6.7 \mathrm{GeV}$

\begin{tabular}{llrr}
\hline Data set & $W, \mathrm{GeV}$ & Number of points & $\chi_{[i]}^{2}$ \\
\hline$[19](\rho)$ & 55 & 10 & 6.5 \\
{$[20](\rho)$} & 73 & 4 & 7.9 \\
{$[21](\rho)$} & 73 & 9 & 36.4 \\
{$[22](\rho)$} & 94 & 10 & 19.0 \\
{$[23](\omega)$} & 80 & 6 & 4.0 \\
{$[24](\phi)$} & 70 & 4 & 1.8 \\
{$[22](\phi)$} & 94 & 4 & 8.6 \\
\hline
\end{tabular}

$g_{\rho \rho \mathrm{P}}(t)=\frac{g_{\rho \rho \mathrm{P}}(0)}{1-b t}$

or

$g_{\rho \rho \mathrm{P}}(t)=g_{\rho \rho \mathrm{P}}(0) e^{b t}$,

we reach the minimum of $\chi^{2}$ at $g_{\rho \rho \mathrm{P}}(0)=7.08 \mathrm{GeV}$ and $b=0.020 \mathrm{GeV}^{-2}$ for both parametrizations. However, the description quality becomes even worse, since the slight decrease of $\chi^{2}$ is overcompensated by the decrease of $N_{D o F}$ due to introduction of extra parameter. Such a result can be interpreted as a manifestation of extremely weak $t$-dependence of $g_{\rho \rho \mathrm{P}}(t)$ and $g_{\omega \omega \mathrm{P}}(t)$ in the diffraction domain.

In view of the fact that the Pomeron coupling to vector mesons is "spin-blind" (SCHC) and comparable to $g_{\pi \pi \mathrm{P}}(0)$ and $g_{K K \mathrm{P}}(0)$, we could expect the existence of some likeness between the Pomeron interactions of vector and scalar mesons and, so, expect a similar weakness of the $t$-dependence of the Pomeron coupling to light scalar mesons, though this expectation should be considered as just an assumption, until a final solution of the above-mentioned secondary Reggeon problem is achieved.

The obtained values of $g_{\rho \rho \mathrm{P}}(0)=g_{\omega \omega \mathrm{P}}(0)$ and $g_{\phi \phi \mathrm{P}}(0)$ allow one to estimate the Pomeron coupling to real photons.

\subsection{High-energy $\gamma p$ scattering}

Owing to the optical theorem: $W^{2} \sigma_{\text {tot }}^{\gamma p}(W)=\operatorname{Im} T_{\gamma p \rightarrow \gamma p}$ $\left(W^{2}, 0\right)$. In the range $W>30 \mathrm{GeV}$, the diagonal fluctuations of photons to mesons $\rho^{0}(770), \omega(782)$, and $\phi(1020)$ provide just about $80 \%$ of $\sigma_{\text {tot }}^{\gamma p}$ (see the dashed line in Fig. 3). Other contributions can be roughly taken into account via introduction of the pole-like term $A(W)=$ $g_{A}(0) g_{p p \mathrm{P}}(0) \pi \alpha_{\mathrm{P}}^{\prime}(0)\left(\frac{W^{2}}{2 s_{0}}\right)^{\alpha_{\mathrm{P}}(0)}$, where $g_{A}(0)=0.007$ $\mathrm{GeV}$, so that the full amplitude is approximated by (the solid line in Fig. 3)

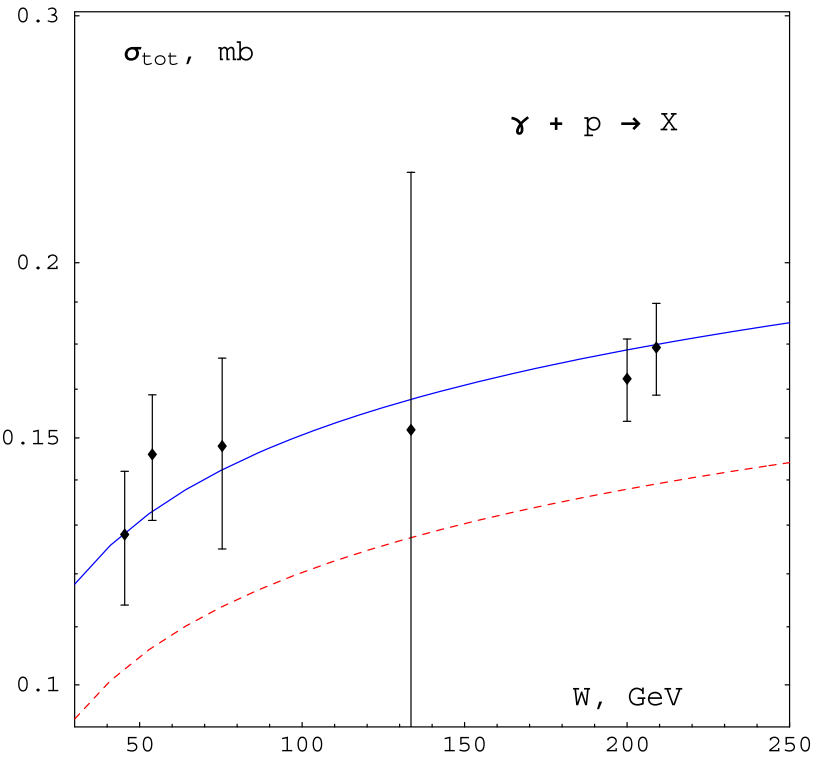

Fig. 3 The total cross sections of $\gamma p$ scattering [5]. The dashed line is the contribution of the fluctuations $\gamma \rightarrow \rho^{0}(770), \omega(782), \phi(1020) \rightarrow$ $\gamma$

$$
\begin{aligned}
& \operatorname{Im} T_{\gamma p \rightarrow \gamma p}\left(W^{2}, 0\right) \\
& \quad \approx \sum_{V=\rho, \omega, \phi}\left[C_{T}^{V}(0)\right]^{2} \operatorname{Im} T_{V p \rightarrow V p}\left(W^{2}, 0\right)+A(W) .
\end{aligned}
$$

The total contribution of absorptive corrections to the imaginary part of the $\rho^{0} p$ scattering forward amplitude is negative. In the interval $40 \mathrm{GeV}<W<210 \mathrm{GeV}$, it does not exceed $16 \%$ of the corresponding Born amplitude. Hence, we do not expect that the underestimation of the Pomeron coupling to photon which emerges under fitting $g_{A}(0)$ to the data is higher than $20 \%$ of this parameter, since the Pomeron interaction of the heavier vector mesons is not expected to be stronger than of the $\rho^{0}(770)$ meson. Thus, we obtain the following estimation of $g_{\gamma \gamma \mathrm{P}}(0)$ :

$$
\begin{aligned}
g_{\gamma \gamma \mathrm{P}}(0)= & \sum_{\substack{V=\rho, \omega, \phi\\
}}\left[C_{T}^{V}(0)\right]^{2} g_{V V \mathrm{P}}(0)+\kappa g_{A}(0) \\
& (1<\kappa<1.2) .
\end{aligned}
$$

\section{The Pomeron decays to light mesons and photons}

Reggeon coupling to light mesons in the diffraction and resonance domains is driven by the same structure functions (see Eqs. (A.3) and (A.4) of the appendix). Although these formulas are initially derived for spinless particles, they can be adapted to vector mesons and photons.

Let us formulate the comparability assumption, which underlies the further discussion:

- The Pomeron coupling to light mesons in the resonance region is comparable or even approximately equal to its 
coupling to the corresponding particles in the diffractive scattering regime. In particular, $\left|g_{h h \mathrm{P}}\left(2 ; M_{\mathrm{P}}^{2}, m_{h}^{2}, m_{h}^{2}\right)\right| \sim$ $\left|g_{h h \mathrm{P}}(0)\right| \equiv g_{h h \mathrm{P}}\left(\alpha_{\mathrm{P}}(0) ; 0, m_{h}^{2}, m_{h}^{2}\right)$, where $M_{\mathrm{P}}$ is the Pomeron ground state mass.

Though this assumption is highly nontrivial (due to the high sensitivity of the resonance partial decay width to the value of its coupling to the decay products), it does not seem to be exceptional. For example, as applied to $\rho$-mesons [2], Eq. (A.4) yields

$\frac{\left|g_{\pi \pi \rho}\left(3 ; M_{\rho_{3}(1690)}^{2}, m_{\pi}^{2}, m_{\pi}^{2}\right)\right|}{\left|g_{\pi \pi \rho}\left(1 ; M_{\rho(770)}^{2}, m_{\pi}^{2}, m_{\pi}^{2}\right)\right|}=1.1 \pm 0.05$.

From the physical standpoint, such a weak $t$-dependence just implies that the corresponding Reggeon perceives pions (and, possibly, some other mesons) as pointlike particles.

Regarding the Pomeron spin-2 resonance decays to pairs of light vector mesons or photons, the comparability assumption supplemented by the above-mentioned SCHC hypothesis (which is valid in the diffraction region) implies the dominance of the only orbital mode: $L=0$. Other orbital modes are related to helicity amplitudes conjugated to the spin-flip vertices in Pomeron exchanges. Hence, they are expected to be dynamically suppressed on the same reasons as the spin-flip amplitudes in the high-energy diffractive scattering. Consequently, in the case of Pomeron interaction, Eq. (A.4) is applicable, as well, to the decays to pairs of vector mesons or photons (of course, the spin-2 resonance decays to pairs of scalar mesons take place in the $L=2$ orbital mode only).

Let us try to describe the $f_{2}(1950)$ resonance observables with the help of the above-stated assumption. For example, the constant related to the Pomeron ground state decay to two real photons of different polarization can be estimated as

$\left|g_{\gamma \gamma \mathrm{P}}\left(2 ; M_{\mathrm{P}}^{2}, 0,0\right)\right|=g_{\gamma \gamma \mathrm{P}}(0)=(39.7 \pm 0.7) \mathrm{MeV}$.

Inserting $M_{\mathrm{P}}=M_{f_{2}(1950)}=(1.944 \pm 0.012) \mathrm{GeV}$ [2] into (A.4), we come to the following result:

$\Gamma_{f_{2}(1950) \rightarrow \gamma \gamma}=(960 \pm 50) \mathrm{eV}$.

In addition, with the help of estimations (8) and (9), we predict

$\Gamma_{f_{2}(1950) \rightarrow \pi^{0} \pi^{0}}=(37 \pm 1) \mathrm{MeV}$,

$\Gamma_{f_{2}(1950) \rightarrow K^{+} K^{-}}=(29 \pm 1) \mathrm{MeV}$,

and, then, in view of $\Gamma_{f_{2}(1950) \rightarrow X}=(472 \pm 18) \mathrm{MeV}$ [2], obtain

$$
\begin{aligned}
\frac{\Gamma_{f_{2}(1950) \rightarrow \gamma \gamma} \Gamma_{f_{2}(1950) \rightarrow \pi^{0} \pi^{0}}}{\Gamma_{f_{2}(1950) \rightarrow X}} & =(75 \pm 5) \mathrm{eV}, \\
\frac{\Gamma_{f_{2}(1950) \rightarrow \gamma \gamma} \Gamma_{f_{2}(1950) \rightarrow K^{+} K^{-}}}{\Gamma_{f_{2}(1950) \rightarrow X}} & =(59 \pm 4) \mathrm{eV},
\end{aligned}
$$

while the corresponding experimental values are $[6,7]$

$$
\begin{aligned}
\frac{\Gamma_{f_{2}(1950) \rightarrow \gamma \gamma} \Gamma_{f_{2}(1950) \rightarrow \pi^{0} \pi^{0}}}{\Gamma_{f_{2}(1950) \rightarrow X}} & =54_{-14}^{+23} \mathrm{eV}, \\
\frac{\Gamma_{f_{2}(1950) \rightarrow \gamma \gamma} \Gamma_{f_{2}(1950) \rightarrow K^{+} K^{-}}}{\Gamma_{f_{2}(1950) \rightarrow X}} & =(61 \pm 2 \pm 13) \mathrm{eV} .
\end{aligned}
$$

The same procedure applied to $f_{2}(2300)$ resonance $\left(M_{f_{2}(2300)}=(2.297 \pm 0.028) \mathrm{GeV}, \Gamma_{f_{2}(2300) \rightarrow X}=(149 \pm\right.$ 40) $\mathrm{MeV}$ [2]) yields the estimation

$$
\frac{\Gamma_{f_{2}(2300) \rightarrow \gamma \gamma} \Gamma_{f_{2}(2300) \rightarrow K \bar{K}}}{\Gamma_{f_{2}(2300) \rightarrow X}}=(1300 \pm 370) \mathrm{eV},
$$

which catastrophically diverges from the Belle data [31]:

$$
\frac{\Gamma_{f_{2}(2300) \rightarrow \gamma \gamma} \Gamma_{f_{2}(2300) \rightarrow K \bar{K}}}{\Gamma_{f_{2}(2300) \rightarrow X}}=3.2_{-0.4-2.2}^{+0.5+1.3} \mathrm{eV} .
$$

This inconsistency allows one to exclude $f_{2}(2300)$ resonance from the candidates for the Pomeron ground state. Unfortunately, analogous data for $f_{2}(2010)$ and $f_{2}(2340)$ resonances [2] are currently unavailable.

\section{Discussion}

Thus, direct propagation of the Pomeron couplings $g_{K K}$ P and $g_{V V \mathrm{P}}$ from the diffraction to resonance region yields a correct estimation of $\Gamma_{f_{2}(1950) \rightarrow \gamma \gamma} \Gamma_{f_{2}(1950) \rightarrow K^{+} K^{-}} / \Gamma_{f_{2}(1950) \rightarrow X}$.

The model and experimental estimations of $\Gamma_{f_{2}(1950) \rightarrow \gamma \gamma}$ $\Gamma_{f_{2}(1950) \rightarrow \pi^{0} \pi^{0}} / \Gamma_{f_{2}(1950) \rightarrow X}$ overlap as well, but the significant systematic uncertainties [6] not included into the presented value (27) make this result not so impressive.

A quite independent argument in favor of our reasoning is the $f_{2}(1950)$ resonance location in the $t-J$ plane (see Fig. 4), which allows a smooth linkage between the linear Chew-Frautschi plot and the nonlinear Regge trajectory of the Pomeron (5) extracted from the nucleon-nucleon scattering data. This coincidence encourages to consider more seriously the possibility of the $f_{2}(1950)$ resonance correspondence to the Pomeron ground state, since other observed $0^{+}\left(2^{++}\right)$resonances do not seem to be so good candidates.

Of course, at present, the discussed coincidences should be qualified just as some noteworthy indications. For a firm conclusion, we need new and, if possible, more accurate data on both the $f_{2}(1950)$ resonance observables and the $\pi p$ and $K p$ scattering at higher energies. Another prediction which should be checked is the opposite polarization of the photons in $f_{2}(1950) \rightarrow \gamma \gamma$ decays which follows from the dominance of the $L=0$ orbital mode predicted above.

In fact, the possible physical consequences of the presumed comparability are much deeper than just a practical feasibility of the Pomeron identification. In the Euclidean domain, the behavior like $\left\{\alpha_{\mathrm{P}}(t)>1, \lim _{t \rightarrow-\infty} \alpha_{\mathrm{P}}(t)=\right.$ $1\}$ is characteristic for glueball Reggeons (for details, see 


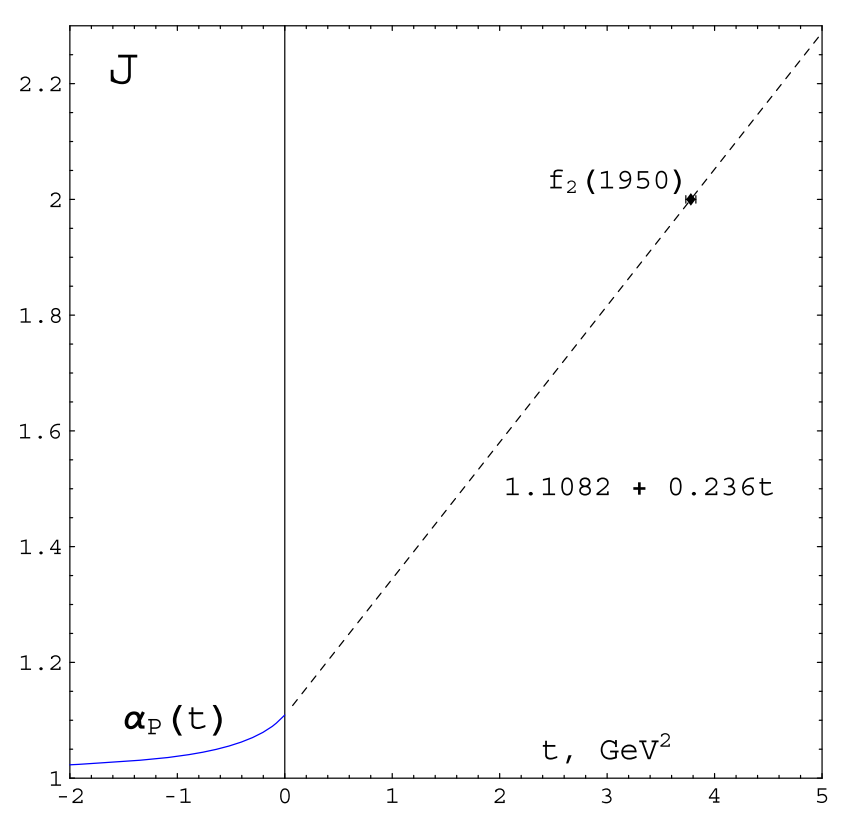

Fig. 4 Expected behavior of the Chew-Frautschi plot for the Pomeron. The solid line is the Pomeron Regge trajectory (5) fitted to the nucleonnucleon scattering data. The dashed line corresponds to $\alpha_{\mathrm{P}}^{\operatorname{lin}}(t)=$ $1.1082+0.236 t$

[10,32-37]). If the assumption is correct, then the Pomeron is expected to keep its glueball nature in the resonance region as well. In other words, if the predictions, regarding the $K \bar{K}, \pi \pi$, and $\gamma \gamma$ decays, are confirmed, we would have enough grounds to consider $f_{2}(1950)$ resonance as not only the Pomeron ground state, but the lightest tensor glueball.

The idea that $f_{2}(1950)$ resonance as a glueball is, certainly, not new. Although its mass is significantly lower than the predictions by lattice QCD [38,39], it is discussed as a glueball candidate from time to time, because of the high value of its decay width [40], and some features of the highenergy $p+p \rightarrow p+f_{2}(1950)+p$ differential cross sections $[41,42]$.

Acknowledgments The author is indebted to his colleagues from the IHEP Division of Theoretical Physics for helpful discussions.

Open Access This article is distributed under the terms of the Creative Commons Attribution 4.0 International License (http://creativecomm ons.org/licenses/by/4.0/), which permits unrestricted use, distribution, and reproduction in any medium, provided you give appropriate credit to the original author(s) and the source, provide a link to the Creative Commons license, and indicate if changes were made.

Funded by SCOAP ${ }^{3}$.

\section{Appendix: Interrelation between Reggeon dynamics in the diffraction and resonance regions}

Let us consider the elastic interaction of two scalar mesons via exchange by single virtual spin- $j$ particle. The contribu- tion of such exchange to the Born amplitude can be represented as

$$
\begin{aligned}
\delta^{\left(j, m_{j}\right)}\left(p_{1}, p_{2}, \Delta\right)= & J_{\alpha_{1} \cdots \alpha_{j}}^{(1)}\left(p_{1}, \Delta\right) \frac{D_{\left(M_{j}\right)}^{\alpha_{1} \cdots \alpha_{j}, \beta_{1} \cdots \beta_{j}}(\Delta)}{m_{j}^{2}-\Delta^{2}} \\
& \times J_{\beta_{1} \cdots \beta_{j}}^{(2)}\left(p_{2},-\Delta\right),
\end{aligned}
$$

where $\frac{D_{\left(M_{j}\right)}^{\alpha_{1} \cdots \alpha_{j}, \beta_{1} \cdots \beta_{j}}(\Delta)}{m_{j}^{2}-\Delta^{2}}$ is the spin- $j$ particle propagator, $m_{j}^{2}=$ $M_{j}^{2}-i M_{j} \Gamma_{j}\left(M_{j}\right.$ and $\Gamma_{j}$ are the mass and decay width of the corresponding resonance), $J_{\alpha_{1} \cdots \alpha_{j}}^{(1,2)}$ are the currents of the scalar mesons, $p_{1}$ and $p_{2}$ are the 4-momenta of the incoming particles, and $\Delta$ is the transferred 4-momentum.

Obviously, in the kinematic range $s \equiv\left(p_{1}+p_{2}\right)^{2} \gg$ $\left\{\left|p_{1,2}^{2}\right|,\left|\Delta^{2}\right|,\left|\left(p_{1,2} \Delta\right)\right|\right\}$ (A.1) transforms into

$$
\begin{aligned}
\delta^{\left(j, m_{j}\right)}\left(p_{1}, p_{2}, \Delta\right) & \frac{g^{(1)}\left(j ; p_{1}^{2}, \Delta^{2},\left(p_{1} \Delta\right)\right) g^{(2)}\left(j ; p_{2}^{2}, \Delta^{2},-\left(p_{2} \Delta\right)\right)}{m_{j}^{2}-\Delta^{2}} \\
& \times\left(\frac{s}{2 s_{0}}\right)^{j}\left[1+O\left(\frac{1}{s}\right)\right],
\end{aligned}
$$

where $g^{(1,2)}$ are the structure functions at the tensor structure $\frac{p_{\alpha_{1}} \cdots p_{\alpha_{j}}}{s_{0}^{j / 2}}$ in the currents $J^{(1,2)}$ and $s_{0} \equiv 1 \mathrm{GeV}^{2}$ is a scale (unit of measurement) characteristic for hadron physics.

The last expression can be used for derivation of the appropriate Reggeon exchange contribution to the Born amplitude for high-energy elastic diffractive scattering (see [1] or, say, the Appendix of [3]):

$$
\begin{aligned}
\delta_{\mathrm{R}}(s, t)= & g_{\mathrm{R}}^{(1)}\left(\alpha_{\mathrm{R}}(t) ; t, m_{1}^{2}, m_{1}^{2}\right) g_{\mathrm{R}}^{(2)}\left(\alpha_{\mathrm{R}}(t) ; t, m_{2}^{2}, m_{2}^{2}\right) \\
& \times \xi\left(\alpha_{\mathrm{R}}(t)\right) \pi \alpha_{\mathrm{R}}^{\prime}(t)\left(\frac{s}{2 s_{0}}\right)^{\alpha_{\mathrm{R}}(t)},
\end{aligned}
$$

where $t \equiv \Delta^{2}<0$, the function $\alpha_{\mathrm{R}}(t)$ is the corresponding Regge trajectory, and $\xi(\alpha)$ is the so-called Reggeon signature factor $\left(\xi(\alpha)=i+\tan \frac{\pi(\alpha-1)}{2}\right.$ for even Reggeons and $\xi(\alpha)=$ $i-\cot \frac{\pi(\alpha-1)}{2}$ for odd Reggeons).

To consider the two-scalar-meson decay of a spin- $j$ resonance state associated with some Reggeon we need to continue (A.1) analytically outside of the $s$-channel physical range. If $p_{1}=-p_{2}, p_{1}^{2}=p_{2}^{2}=m_{a}^{2},\left(p_{1}-\Delta\right)^{2}=$ $\left(p_{2}+\Delta\right)^{2}=m_{b}^{2}$, and $\Delta^{2} \rightarrow M_{j}^{2}>\left(m_{a}+m_{b}\right)^{2}$, then, in view of the general properties of $D_{\left(M_{j}\right)}^{\alpha_{1} \cdots \alpha_{j}, \beta_{1} \cdots \beta_{j}}(\Delta)$ at $\Delta^{2}=M_{j}^{2}$ (the transversality with respect to $\Delta_{\alpha_{k}}(k=1, \ldots, j)$ and the tracelessness with respect to any pair of Lorentz indices like $\left\{\alpha_{i} \alpha_{j}\right\}$ and $\left\{\beta_{i} \beta_{j}\right\}$ ), we obtain the following expression for the resonance partial decay width:

$$
\Gamma_{\mathrm{R} \rightarrow a+b}^{(j)}=\frac{\lambda^{1 / 2}\left(M_{j}^{2}, m_{a}^{2}, m_{b}^{2}\right)}{16 \pi M_{j}^{3}}\left|A_{\mathrm{R} \rightarrow a+b}^{(j)}\right|^{2}
$$




$$
\begin{aligned}
= & \frac{\lambda^{1 / 2}\left(M_{j}^{2}, m_{a}^{2}, m_{b}^{2}\right)}{16 \pi M_{j}^{3}} \frac{\left|g_{a b \mathrm{R}}\left(j ; M_{j}^{2}, m_{a}^{2}, m_{b}^{2}\right)\right|^{2}}{(2 j+1) s_{0}^{j}} \\
& \times p_{\alpha_{1}} \cdots p_{\alpha_{j}} p_{\beta_{1}} \cdots p_{\beta_{j}} D_{\left(M_{j}\right)}^{\alpha_{1} \cdots \alpha_{j}, \beta_{1} \cdots \beta_{j}}(\Delta) \\
= & \frac{(j !)^{2} \lambda^{j+1 / 2}\left(M_{j}^{2}, m_{a}^{2}, m_{b}^{2}\right)}{16 \pi 2^{j}(2 j+1) ! M_{j}^{2 j+3} s_{0}^{j}}\left|g_{a b \mathrm{R}}\left(j ; M_{j}^{2}, m_{a}^{2}, m_{b}^{2}\right)\right|^{2},
\end{aligned}
$$

where $\lambda(x, y, z) \equiv x^{2}+y^{2}+z^{2}-2 x y-2 y z-2 x z$.

\section{References}

1. P.D.B. Collins, An Introduction to Regge Theory and High Energy Physics (Cambridge University Press, Cambridge, 1977)

2. Particle Data Group, http://pdg.lbl.gov/2015/tables/ rpp2015-sum-mesons.pdf

3. A.A. Godizov, Eur. Phys. J. C 75, 224 (2015)

4. A. Donnachie, P.V. Landshoff, Phys. Lett. B 296, 227 (1992)

5. Particle Data Group, http://pdg.lbl.gov/2015/hadronic-xsections/ hadron.html

6. The Belle Collaboration (S. Uehara et al.), Phys. Rev. D 79, 052009 (2009)

7. The Belle Collaboration (K. Abe et al.), Eur. Phys. J. C 32, 323 (2004)

8. A. Donnachie, P.V. Landshoff, Nucl. Phys. B 267, 690 (1986)

9. S. Donnachie, G. Dosch, P. Landshoff, O. Nachtmann, Pomeron Physics and $Q C D$ (Cambridge University Press, Cambridge, 2002)

10. P.D.B. Collins, P.J. Kearney, Z. Phys. C 22, 277 (1984)

11. NA22 Collaboration (M. Adamus et al.), Phys. Lett. B 186, 223 (1987)

12. J. Kwiecinski, Phys. Rev. D 26, 3293 (1982)

13. R. Kirschner, Z. Phys. C 67, 459 (1995)

14. A.V. Barnes et al., Phys. Rev. Lett. 37, 76 (1976)
15. O.I. Dahl et al., Phys. Rev. Lett. 37, 80 (1976)

16. V.D. Apokin et al., Nucl. Phys. B 255, 253 (1985)

17. ZEUS Collaboration (M. Derrick et al.), Z. Phys. C 63, 391 (1994)

18. ZEUS Collaboration (M. Derrick et al.), Z. Phys. C 69, 39 (1995)

19. H1 Collaboration (S. Aid et al.), Nucl. Phys. B 463, 3 (1996)

20. ZEUS Collaboration (M. Derrick et al.), Z. Phys. C 73, 253 (1997)

21. ZEUS Collaboration (J. Breitweg et al.), Eur. Phys. J. C 2, 247 (1998)

22. ZEUS Collaboration (J. Breitweg et al.), Eur. Phys. J. C 14, 213 (2000)

23. ZEUS Collaboration (M. Derrick et al.), Z. Phys. C 73, 73 (1996)

24. ZEUS Collaboration (M. Derrick et al.), Phys. Lett. B 377, 259 (1996)

25. ZEUS Collaboration (S. Chekanov et al.), Nucl. Phys. B 695, 3 (2004)

26. ZEUS Collaboration (S. Chekanov et al.), Nucl. Phys. B 718, 3 (2005)

27. ZEUS Collaboration (S. Chekanov et al.), PMC Phys. A 1, 6 (2007)

28. H1 Collaboration (F.D. Aaron et al.), JHEP 1005 (2010) 032

29. J.J. Sakurai, Currents and Mesons (The University of Chicago Press, Chicago, 1969)

30. V.A. Petrov, Mod. Phys. Lett. A 30, 1550164 (2015)

31. The Belle Collaboration (S. Uehara et al.), Prog. Theor. Exp. Phys. $2013123 \mathrm{C} 01$

32. F.E. Low, Phys. Rev. D 12, 163 (1975)

33. S. Nussinov, Phys. Rev. D 14, 246 (1976)

34. D. Heckathorn, Phys. Rev. D 18, 1286 (1978)

35. H. Cheng, T.T. Wu, Phys. Rev. Lett. 22, 666 (1969)

36. R. Kirschner, L.N. Lipatov, Z. Phys. C 45, 477 (1990)

37. A.A. Godizov, V.A. Petrov, JHEP 0707, 083 (2007)

38. Y. Chen et al., Phys. Rev. D 73, 014516 (2006)

39. E. Gregory et al., JHEP 1210, 170 (2012)

40. F. Brünner, D. Parganlija, A. Rebhan, Phys. Rev. D 91, 106002 (2015)

41. WA102 Collaboration (D. Barberis et al.), Phys. Lett. B 474, 423 (2000)

42. A. Kirk, Phys. Lett. B 489, 29 (2000) 